\title{
Analysing Audience Response to Performing Events
}

\section{A Web Platform for Interactive Exploration of Physiological Sensor Data}

\author{
Thomas Röggla \\ CWI \\ Science Park 123 \\ Amsterdam, Netherlands \\ t.roggla@cwi.nl
}

\author{
Chen Wang \\ CWI \\ Science Park 123 \\ Amsterdam, Netherlands \\ c.wang@cwi.nl
}

\author{
Pablo Cesar \\ CWI \\ Science Park 123 \\ Amsterdam, Netherlands \\ p.s.cesar@cwi.nl
}

\begin{abstract}
This paper presents a web interface for the exploration of audience response to a performing arts event. The platform temporally synchronises data obtained from physiological sensors with video recordings. More concretely, it is geared towards people from the creative industry, e.g. theatre directors, who want to gain deeper insights into how audiences perceive their performances. The platform takes the raw data from sensors and corresponding video recordings and visualises both synchronised in a more digestible manner. This document presents the major features of the application and explains the reasoning behind its various visualisations and interactive capabilities and how it can benefit performing artists.
\end{abstract}

\section{Categories and Subject Descriptors}

H.5.m. [Information Interfaces and Presentation (e.g. HCI)]: multimedia information systems, user interfaces

\section{General Terms}

Experimentation, Human Factors

\section{Keywords}

Physiological Sensors, Visualisation, Web Technologies

\section{INTRODUCTION}

The field of physiological sensors has become an active area of research because it allows researchers to measure parameters of the human physiology and to link them to psychological concepts such as emotion, attention or engagement [3]. The data obtained from these sensors is comprised of streams of numbers, which in themselves are rather meaningless. It takes domain knowledge as well as mathematical tools to interpret this data and assign it some meaning.

Our previous research in the context of theatre performances, where audience members were equipped with GSR

Permission to make digital or hard copies of part or all of this work for personal or classroom use is granted without fee provided that copies are not made or distributed for profit or commercial advantage and that copies bear this notice and the full citation on the first page. Copyrights for third-party components of this work must be honored. For all other uses, contact the Owner/Author.

Copyright is held by the owner/author(s).

MM'15 October 26-30, 2015, Brisbane, Australia

ACM 978-1-4503-3459-4/15/10.

DOI: http://dx.doi.org/10.1145/2733373.2807976
(Galvanic Skin Response) sensors, has shown that people who might profit from such measurements, namely performers or directors, often have trouble interpreting the results. What is presented in this paper is a web-based tool which allows the user to play back video recordings of performances where audience members were wearing physiological sensors. The platform takes in the raw sensor data, which needs to be in temporal synchronisation with the video recordings and presents it in the form of various visualisations, making it easier to interpret and understand. The data and video recordings used for testing the web application were taken from our previous experiments [4].

The main design goals behind the platform, besides enabling easier interpretation of sensor data, was to foster collaboration by allowing multiple users, e.g. theatre directors, to annotate sections of the video recording. To make the experience more interactive, the user can jump around in the recording and see the performance from different camera angles to gain a more complete picture of the audience's reaction to a piece, thereby providing artists with a better user experience in the most unobtrusive way.

The contribution of this tool is to provide creative people with a means of post-performance analysis of audience reaction. More concretely, users of the application can seek to random parts of the performance and observe the stage and the audience in the video and cross-reference it with the sensor data. Different from existing tools like $[1,3]$, we focus on user reaction and engagement and provide a way for performing artists to assess it in a more intuitive way, whereas previous work did not include sensor data at all or only did so in a laboratory setting.

\section{APPROACH}

To achieve this, we have implemented a web interface taking advantage of web technologies including HTML5 video playback and interactive charts using SVG and JavaScript. The application is backed by a web server serving the content and a database holding the sensor data. This makes sure that the application works anywhere the user has access to the Internet via a reasonably modern web browser. Furthermore, in this way, the data is kept in a centralised location, which enables several users to interact with the application at once. To protect access to the data, each user has to log in with a personal password.

While we did set a handful of basic requirements for the application ourselves, such as video playback and the possibility to switch between camera angles, the main bulk of the features was developed using direct input from artists. We 
followed a human-centered approach, involving users from the very beginning in one-on-one interviews and focus group discussions. Performing artists were also asked in the final evaluation process, which involved them testing and rating it using the CSI (Creativity Support Index) defined in [2], which gives us a comparable value for different factors of creativity, such as Collaboration, Enjoyment, Exploration, Expressiveness, Immersion, and Results Worth Effort.

\subsection{Video Page}

Figure 1 illustrates the top part of the video page of the application, which shows the video playback window, simple playback controls, controls for changing the camera angles and the seek bar, with which the user can jump to arbitrary positions in the timeline.

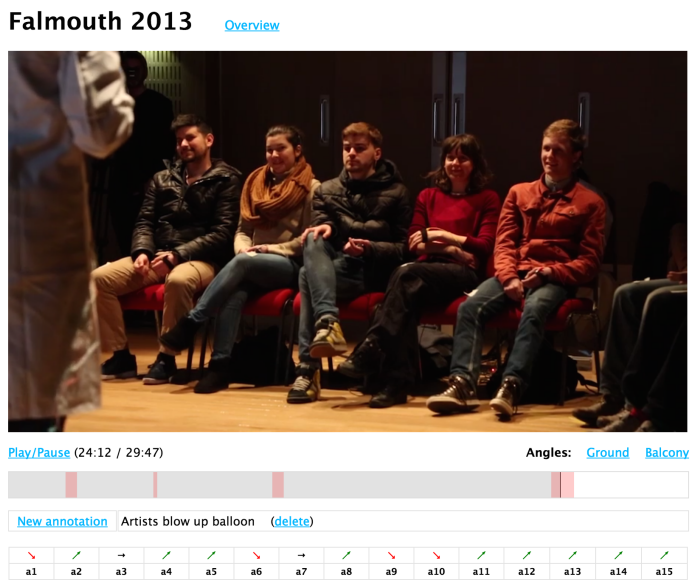

Figure 1: Video page of the application, playing back a video in a browser window

The red sections overlaid on certain parts of the timeline represent textual annotations which have been left by users. An annotation is displayed for as long as the timeline scrubber intersects with the red section. In the figure, the timeline scrubber is currently positioned over such an annotation and its content is displayed in the area below the timeline. This control field can also be used to add new annotations or delete the current one. Artists saw this annotation mechanism as a very important feature, as it allows them to tie comments and notes to specific points in time.

The elements below the annotation controls are devoted to an individual audience member's sensor measurements at the current point in the timeline. The table contains one entry for each audience member, while the arrows represent whether the sensor response is currently on the rise, decline or if it stays within a certain threshold. This is meant to give an indication whether the person wearing the sensor is currently getting more engaged with the performance, indicated by a green arrow pointing up, or if, on the other extreme, starts losing interest, which is indicated by a red arrow pointing down.

Figure 2 shows the remaining part of the video page. It contains detailed line charts showing the evolution of the sensor response for the duration of the entire performance. The user can view an individual's response curve by selecting a participant's name in the table in Figure 1. The averaged sensor response of the entire audience is always displayed be-

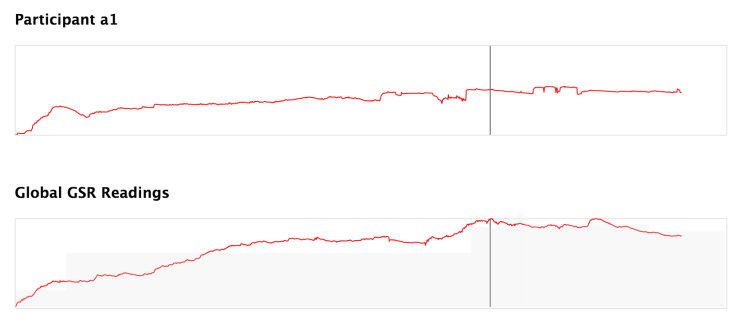

Figure 2: Lower part of the video page, with individual response curve and an average for the whole audience.

low that. These line charts double as seek bars, allowing the user to exactly pinpoint a location and see what happening at that specific point in time by clicking on it.

\section{CONCLUSION}

In the evaluation process, we had testers use the application and rate it using CSI, giving an indication about how well it supports the creative process. In the results, the testers rated the factors of Exploration and Collaboration the highest, which largely coincides with our design goals.

After these tests and more discussions with potential end users, which supplied us with another round of feedback, we were able to conclude that the tool could be a valuable tool for artists to gauge an audience's reaction to a performance in an easy way. More specifically, it gives them a means to see which parts of a performance are perceived well and which parts still might have room for improvement. The annotation system was added with this use case in mind.

Finally, one should also note that even though the example of the application given in this paper displays GSR data, it can be used to visualise any type of sensor data from any number of users, as long as it is quantifiable, i.e. it is comprised of a stream of numbers and can be reasonably displayed on a temporal axis. The application is designed in such a way that it could display data from multiple sources at once, provided that they are in temporal synchrony with video recordings. The example also uses two video recordings, but in practice, the application can support any number of recordings.

While the results from the testers were positive and largely coincided with our expectations, we believe that the tool still requires more real-world testing to really prove its usefulness and collect more valuable feedback to improve user experience, usability and add potential new features.

\section{REFERENCES}

[1] D. Cabral et al. Evaluation of a multimodal video annotator for contemporary dance. In Proceedings of the International Working Conference on Advanced Visual Interfaces, AVI '12, pages 572-579, 2012.

[2] E. Cherry et al. Quantifying the creativity support of digital tools through the creativity support index. $A C M$ Trans. Comput.-Hum. Interact., 21(4):21:1-21:25, 2014.

[3] C. Latulipe et al. Love, hate, arousal and engagement: exploring audience responses to performing arts. In Proceedings of CHI, pages 1845-1854, 2011.

[4] C. Wang et al. Sensing a live audience. In Proceedings of CHI, CHI '14, pages 1909-1912, 2014. 